\title{
Analytical study of flexible stimulation waveforms in muscle fatigue reduction
}

\author{
E. Noorsal, S. Z. Yahaya, Z. Hussain, R. Bouldville, M. N. Ibrahim, Y. Mohd Ali \\ Faculty of Electrical Engineering, Universiti Teknologi MARA Pulau Pinang, Malaysia
}

\begin{tabular}{l}
\hline \hline Article Info \\
\hline Article history: \\
Received Apr 5, 2019 \\
Revised Sep 10, 2019 \\
Accepted Sep 27, 2019 \\
\hline
\end{tabular}

\section{Keywords:}

Flexible stimulation waveform Functional electrical stimulator (FES)

Hemiplegic

Humanoid muscle model

Muscle fatigue

Neuromuscular

\begin{abstract}
This paper presents the analytical study of flexible stimulation waveforms in muscle fatigue reduction for functional electrical stimulator (FES)-assisted hemiplegic muscle activities. The major challenge of muscle contraction induced by FES is early muscle fatigue which greatly limits activities such as FES-assisted standing and walking. The fixed stimulation pattern applied on a same motor unit has resulted the motor unit to be overworked and fatigue easily. Therefore, in this work, the stimulus parameters, which include the pulse width duration and the frequency were varied to create a few flexible stimulation waveforms using MATLAB/Simulink. The pulse width duration was modulated from $100 \mu \mathrm{s}-500 \mu$ s to generate five types of flexible stimulation waveforms such as Rectangular, Trapezoidal, Ramp Up, Ramp Down and Triangular. Concurrently, a few ranges of stimulus frequency were also used, which include $20 \mathrm{~Hz}, 30 \mathrm{~Hz}$ and $50 \mathrm{~Hz}$. The generated flexible stimulation waveforms were applied onto a humanoid muscle model to investigate and analyse the muscle output response and early muscle fatigue reduction. From the conducted simulation results and analyses, it was observed that flexible stimulation waveforms such as Triangular, Ramp Up and Ramp Down could reduce early muscle fatigue phenomenon by having lower average of negative slope, in the range of 0.012 to 0.013 for the muscle fitness. In contrast, the Rectangular and Trapezoidal shapes were found to have higher negative slope of muscle fitness in the range of 0.028 to 0.031 . The Ramp Down shape was found to have the lowest average of negative slope (0.012) while Rectangular was found to have the highest average of negative slope (0.031). Therefore, it can be concluded that flexible stimulation waveforms such Ramp Down, Ramp Up and Triangular shapes could reduce early muscle fatigue phenomenon with Ramp Down shape having the highest muscle fatigue reduction.
\end{abstract}

Copyright $@ 2020$ Institute of Advanced Engineering and Science. All rights reserved.

\section{Corresponding Author:}

Emilia Noorsal,

Faculty of Electrical Engineering, Universiti Teknologi MARA Pulau Pinang,

Permatang Pauh, 13500, Malaysia.

Email: emilia.noorsal@uitm.edu.my

\section{INTRODUCTION}

To date, several external and implantable functional electrical stimulation (FES) devices have been developed for neuromuscular applications to restore function to paralysed muscles and limbs, bowel and bladder excretion and the respiratory system [1-5]. The FES triggers an action potential in the neural axons by transferring an electrical stimulation charge into neural tissue. The applied stimulus charge artificially depolarizes the nerve membrane and generates an action potential. It is important to note that the choice of stimulation wave shapes and patterns has a direct effect on the physiological response generated by the stimulus $[6,7]$. Therefore, to achieve a desired outcome of stimulation, an optimum waveform is required. 
The FES involves artificially inducing a current in specific motor neurons to generate muscle contractions [8,9]. The neurons receives a series of short electrical pulses that are delivered using electrodes [10]. These electrodes can be transcutaneous (placed on the skin surface), percutaneous (placed within a muscle), epimysia (placed on the surface of the muscle), or cuff (wrapped around the nerve that innervate the muscle of interest) [10]. The tension produced in electrically stimulated muscle depends on the intensity and frequency of stimulation. The stimulation intensity is a function of the total charge transferred to he muscle, which depends on the pulse amplitude, duration and frequency as well as the shape of the pulse train. These muscle contractions can be adjusted by stimulating one or more muscles that will create torque at the joint of the muscle.

Unfortunately, one of the major limitations in muscle contractions induced by FES is early or rapid muscle fatigue, which greatly limits FES-assisted activities such as standing and walking. Reduction in muscle force due to the rapid fatigability of paralysed muscle with electrical excitation in FES device can lead to instability, poor control and limited walking distances [11]. Thus, applications of electrical stimulation for restoration of functional movements such as standing, gait, and grasp have always been hindered by the rapid fatigue of stimulated muscle $[12,13]$. One of the main reasons for this early muscle fatigue is the inefficiency in FES stimulation system [6]. The two main limitations in FES system are reverse order recruitment (non-physiological recruitment) and bias synchronous recruitment of motor neurons for muscle contraction. This is different from the central nervous system (CNS), which triggers action potentials asynchronously and allow the motor unit to rest. In physiological muscle contraction, the smallest motor axons (high fatigue-resistant) are recruited first and the largest motor axons (least fatigue-resistant) are recruited last for smooth muscle contraction.

Motivated by the discrepancies of the FES system, many stimulation techniques have been designed and developed to help alleviate the problem of early muscle fatigue in FES-induced muscle contraction. Among the main stimulation intensity parameters are the voltages and current level, pulse shape, pulse width and pulse frequency. The amplitude and current defines the strength of stimulation or how strong is the force generated to the muscle. The stimulation frequency mainly controls twitching of the muscle. As the stimulation frequency increases, the stimulated muscle will get fatigue fasters which inadvertently limit the muscle training duration [14]. Therefore, in [11] the stimulation frequency had been reduced to decrease fatigue of the activated muscle fatigue. Graupe et. al [13] had employed random modulation of FES stimulation parameters to reduce muscle fatigue. By randomly modulating the pulse frequency, amplitude and width, the resulting firing rate and level of recruitment of motor units would vary over time. Other methods of fatigue reduction have practical limitation such as double stimulation and sequential stimulation. Double stimulation used by Routh et al., had demonstrated both a positive and negative effect on the fatigue time depending on the test conditions and protocol. An alternative technique employed by Karu et al. [12] to combat early muscle fatigue was by implementing $\mathrm{N}$-let (a set of $\mathrm{N}$ closely spaced pulses) stimulation method. The advantage of using optimized N-let stimulation trains technique is it greatly increases the ability of a stimulated muscle to sustain force during an isometric contraction when compared to conventional FES which used single pulse train stimulation.

Despite several efforts of using different stimulation strategies, it is important to note that most of the stimulation waveforms are restricted to rectangular pulse shape and fatigue continues to be a limiting factor in the wide-spread application of FES techniques in rehabilitation practice. Therefore, there is still much room for improvement in the FES device to produce efficient stimulation technique that can optimize the muscle torque output and subsequently reduce the effect of early muscle fatigue. Recently, various experimental and clinical studies have demonstrated that different neuronal-cell targets require different threshold levels, different types of stimulation waveforms for excitation and different stimulation strategies. Additionally, the benefits of flexible stimulation waveforms for neural prosthesis applications were demonstrated in various publications [7, 15-18]. Thus, a highly efficient and flexible stimulator that has great flexibility in stimulation waveform generation is essentially required for robust and universal applications. Some of the benefits of flexible stimulation waveforms that are mainly related to neuromuscular application, stimulation efficiency and safety are summarized as follows: (i) Arbitrary pulse shape such as quasitrapezoidal [18] and triangular pulse shape [19] were found to provide good selectivity to stimulate a specific population of nerve fibres (fibre diameter or spatial selectivity) without activating the neighbouring and unwanted nerve. (ii) High frequency rectangular biphasic pulse train in range of 100 to $600 \mathrm{~Hz}$ were found to be able block large diameter fibres into firing action potentials [15]. (iii) Non-rectangular shape, such as gaussian, linear or exponential decrease, was the most efficient pulse shape that maximized the charge injection capacity of the electrode with the lowest threshold charge for neural excitation [17]. (iv) A linear decreasing ramp was found to provide the best charge injection for all pulse widths ranging from 0.02 to $0.5 \mathrm{~ms}$ [17]. (v) The exponential waveform, for a stimulation time duration between $100 \mu \mathrm{s}$ and $5 \mathrm{~ms}$, was found by [20] to be the safest and most energy-optimal waveform with lower power consumption, 
low charge injection and more reduced product concentration deviations. (vi) Gaussian and sinusoidal stimulus wave shapes were observed by [21] to deliver much smaller peak current densities than a square stimulus shape.

In summary, based on the diversity of neural stimulator requirements together with numerous research studies and findings on stimulation efficiency, having flexible stimulation waveforms is advantageous for different degrees of tissue degeneration, for efficient stimulation and for maximizing the application range of the FES. Experimental studies conducted by several researchers have shown that several non-rectangular shapes, such as Gaussian, linear decrease and exponential increase, require less energy to trigger the action potential and thus provide efficient stimulation.

Therefore, the objective of this research work is to investigate the relationship of muscle output response with flexible stimulation waveforms in FES modelling system using humanoid muscle model. The stimulus parameters, which include the pulse width duration and frequency were varied to generate the flexible stimulation waveforms using MATLAB/Simulink. Five types of flexible waveforms were tested onto a humanoid muscle model namely Rectangular, Trapezoidal, Ramp up, Ramp down and Triangular. The muscle output response was evaluated and analysed over a period of 60 s to observe the early muscle fatigue reduction when flexible stimulation waveforms were injected onto the muscle model.

\section{RESEARCH METHOD}

The studies conducted in this research involve with the development of flexible stimulation waveforms, testing the generated flexible stimulation waveforms onto a humanoid muscle model and finally analysed the muscle response with respect to the flexible stimulation waveforms as illustrated in the Figure 1 flowchart. Five flexible stimulation waveforms were developed using MATLAB/Simulink by varying the pulse width from $100 \mu \mathrm{s}-500 \mu \mathrm{s}$. The modulation of stimulation pulse width resulted in five types of flexible stimulation waveforms such as Rectangular, Trapezoidal, Ramp Up, Ramp Down and Triangular. Additionally, three stimulation frequencies were used namely $20 \mathrm{~Hz}, 30 \mathrm{~Hz}$ and $50 \mathrm{~Hz}$. Table 1 lists detail stimulus parameter settings such as pulse width, frequency and stimulation duration for the overall test setup. Each waveform has different characteristic and can be distinguished by the waveforms shape, pulse width and frequencies. To test the performance of the flexible stimulation waveform, a physiologically based muscle model (muscle activation dynamic) was constructed. This muscle activation dynamic model enables the analysis of muscle activation, which include the muscle fatigue characteristic.

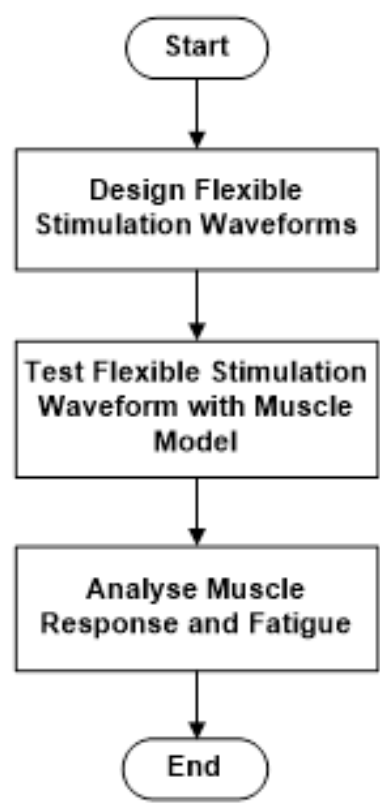

Figure 1. Research flow methodology 
Table 1. Flexible stimulation waveforms with corresponding stimulus

\begin{tabular}{|c|c|c|c|c|c|c|}
\hline \multirow{2}{*}{$\begin{array}{c}\text { Flexible } \\
\text { Waveform }\end{array}$} & \multirow{2}{*}{$\begin{array}{l}\text { Pulse Width } \\
\text { Modulation }\end{array}$} & \multicolumn{2}{|c|}{ Stimulation Duration } & \multicolumn{3}{|c|}{ Frequency } \\
\hline & & $2 \mathrm{~s} \mathrm{ON}$ & $\begin{array}{l}2 \mathrm{~s} \mathrm{ON}, 3 \mathrm{~s} \\
\text { OFF for } 60 \mathrm{~s}\end{array}$ & $20 \mathrm{~Hz}$ & $30 \mathrm{~Hz}$ & $50 \mathrm{~Hz}$ \\
\hline \multirow[t]{5}{*}{ Rectangular } & $100 \mu \mathrm{s}$ & I & 1 & I & I & I \\
\hline & $200 \mu \mathrm{s}$ & I & I & l & I & I \\
\hline & $300 \mu \mathrm{s}$ & / & I & l & l & / \\
\hline & $400 \mu \mathrm{s}$ & I & I & I & l & I \\
\hline & $500 \mu \mathrm{s}$ & I & I & I & l & I \\
\hline Trapezoidal & $100-500 \mu \mathrm{s}$ & l & I & & l & \\
\hline Ramp Up & $100-500 \mu \mathrm{s}$ & / & I & & I & \\
\hline Ramp Down & $100-500 \mu \mathrm{s}$ & I & I & & l & \\
\hline Triangular & $100-500 \mu \mathrm{s}$ & I & 1 & & I & \\
\hline
\end{tabular}

\subsection{Flexible stimulation waveform model}

In most application of FES, non-invasive type of intervention using surface electrode is used. This is due to its simple preparation and adequately efficient to achieve the objective of the FES application. In this study, the flexible stimulation waveforms were designed by considering the issues related with the surface stimulation such as synchronous activation of muscle fibre and penetration via other tissues before reaching the targeted muscle. MATLAB/Simulink software was used to design the 5 types of waveforms and to test the generated waveforms with a muscle model. Figure 2 shows the flexible stimulation waveforms model that was developed using MATLAB/Simulink.

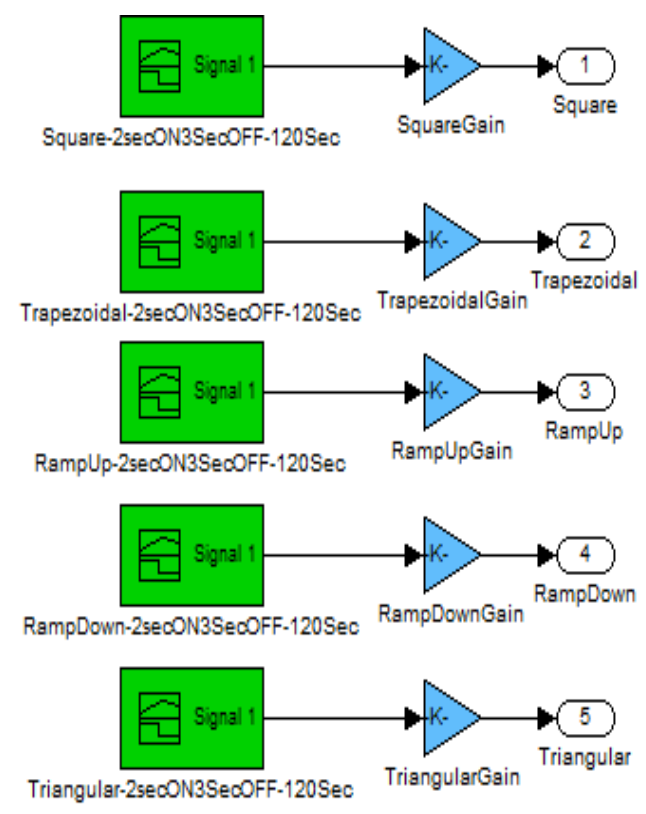

Figure 2. Flexible stimulation waveforms model

In generating the individual waveforms, a Signal Builder function was used. As listed in Table 1, all waveforms share a common parameter that is $2 \mathrm{~s}$ ON and $3 \mathrm{~s}$ OFF continuously for a duration of $60 \mathrm{~s}$. This means, individual waveforms will have perfect shape for every $2 \mathrm{~s}$ and re-appear every $5 \mathrm{~s}$. Within the $2 \mathrm{~s}$ $\mathrm{ON}$, the stimulation pulse width will be modulated to form a complete shape of waveforms. The duration of 60s stimulation period ensures continuous production of waveforms for 60s.

The waveforms peak value is determined by the user and normally allowed to be a maximum of $500 \mu$ s according to the standard practice for FES [22]. Each Signal Builder output is connected with a Gain function to enable the user to set the required peak value of stimulation pulse width. Figure 3 depicts the designed flexible stimulation waveforms model output waveforms at maximum peak of $250 \mu$ s and simulated for $60 \mathrm{~s}$. 


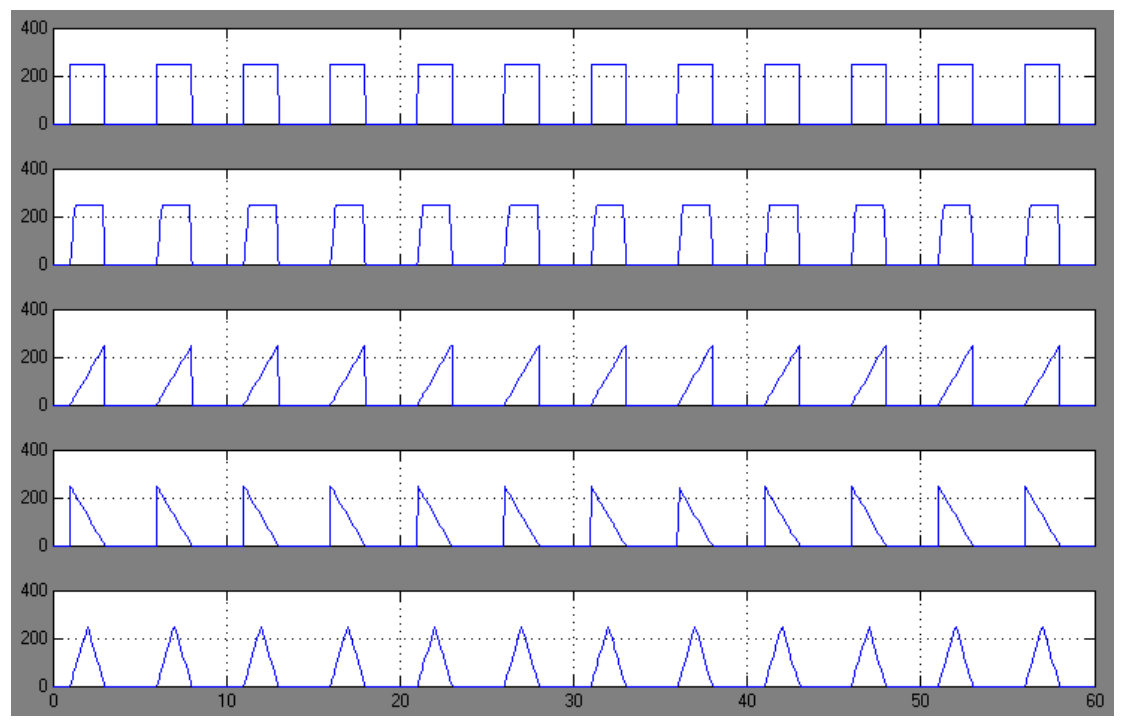

Figure 3. Flexible stimulation waveforms model output

\subsection{Flexible stimulation waveform with muscle model}

The muscle model structure as formulated by Riener \& Fuhr [22] consists of three main sections which are the muscle activation dynamics, contraction dynamics and body segmental dynamics. In this study, only part of the muscle model, which is the muscle activation dynamics was constructed in MATLAB/Simulink and is shown in Figure 4.

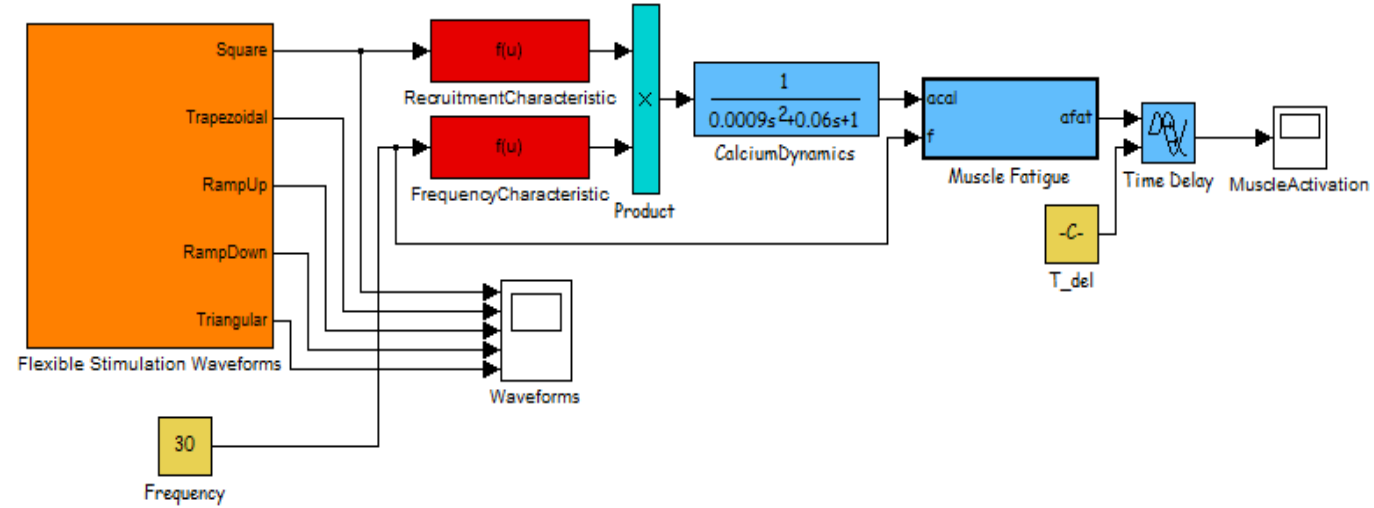

Figure 4. Flexible stimulation waveform with muscle model

In this work, the muscle activation model proposed by Riener and Fuhr [22] was used as shown in Figure 4. The muscle activation model consists of four main components; Recruitment Characteristic, Frequency Characteristic, Calcium Dynamics and Muscle Fatigue. In calculating the muscle activation level, the muscle activation dynamics requires several input parameters. The input to Recruitment Characteristic model is the stimulation pulse width. Thus, it is connected to the flexible stimulation waveforms, which provide the stimulation pulse width modulation. The Recruitment Characteristic model describes the percentage of normalized motor unit of the muscle that is activated and computed as a function of stimulation pulse width. For the Frequency Characteristic model, the input is the stimulation frequency that is the set frequency of the stimulation received by the muscle. The Frequency Characteristic model describes the normalized amount of activation in a single motor unit. This function has been introduced to capture the force-frequency characteristic of artificially stimulated muscle. The output product of Recruitment Characteristic model and Frequency Characteristic output became the input to the Calcium Dynamic model. The Calcium Dynamic, which is modelled using two first order transfer functions produce a non-fatiguing muscle activation. This non-fatiguing muscle activation is then applied with fatigue by Muscle Fatigue model to produce the muscle activation level. 
The muscle activation dynamic is used to observe the activation output as the flexible stimulation waveforms is applied to the input. In the overall structure of muscle model by Riener \& Fuhr [22], the output of muscle activation dynamic became the input to the muscle contraction dynamics to produce the muscle force. Subsequently, the product of muscle force with moment arm will result with active joint moment (torque). The overall joint torque is produced by taking into consideration the passive joint torque generated passively from the movement. Detail explanation, equations and parameters for the muscle model can be obtained in $[22,23]$.

\subsection{Stimulation strategy of flexible waveform for muscle fatigue analyses}

For muscle fatigue analyses purpose, the generated flexible stimulation waveforms were tested in four different stages, which are listed as follows:

a. Single Rectangular stimulation waveform with pulse width duration ranging from $100 \mu \mathrm{s}-500 \mu \mathrm{s}$ and three frequency settings $(20 \mathrm{~Hz}, 30 \mathrm{~Hz}, 50 \mathrm{~Hz})$,

b. Pulse train of Rectangular stimulation waveform with pulse width duration ranging from $100 \mu \mathrm{s}-500 \mu \mathrm{s}$ and three frequency settings $(20 \mathrm{~Hz}, 30 \mathrm{~Hz}, 50 \mathrm{~Hz})$,

c. Single flexible stimulation waveform using pulse width modulation from $100 \mu$ s $-500 \mu$ s and stimulation frequency of $30 \mathrm{~Hz}$. The pulse width modulation resulted in flexible waveforms generation such as Trapezoidal, Ramp Up, Ramp Down and Triangular shapes. For Rectangular shape the pulse width of 500 $\mu$ s was used.

d. Pulse train of flexible stimulation waveform using pulse width modulation from $100 \mu \mathrm{s}-500 \mu \mathrm{s}$ and stimulation frequency of $30 \mathrm{~Hz}$. Similar to single flexible waveforms, five different types of flexible stimulation waveforms were generated, which include Rectangular, Trapezoidal, Ramp Up, Ramp Down and Triangular shapes.

Initially, only single Rectangular shape was tested to observe the output response of muscle activation for different pulse width and frequency settings. Secondly, the pulse train of Rectangular shape was used to observe the muscle activation response for a duration of 60 s stimulation period. Thirdly, single flexible waveform shapes were used to compare the response of muscle activation among the five generated flexible stimulation waveforms. Finally, the pulse train of flexible stimulation waveforms were tested to observe the muscle fitness over a duration of 60 s stimulation period. Details on the selection of parameter settings for each stage will be further discussed in results and discussion section.

\section{RESULTS AND ANALYSIS}

In this section, the simulation results obtained are discussed. As discussed in previous section, five different types of flexible stimulation waveforms (Rectangular, Trapezoidal, Ramp Up, Ramp Down and Triangular) were developed by modulating the pulse width duration from $100 \mu \mathrm{s}-500 \mu$ s with three different values of frequencies $20 \mathrm{~Hz}, 30 \mathrm{~Hz}, 50 \mathrm{~Hz}$. The generated flexible stimulation waveforms were tested onto a humanoid muscle model, in which the muscle output response and muscle fitness were measured and analysed. As mentioned earlier, the generated flexible stimulation waveforms were tested in four different stages; which include (i) single Rectangular stimulation waveform (ii) pulse train of Rectangular stimulation waveform (iii) single flexible stimulation waveform, and (iv) pulse train of flexible stimulation. Therefore, the following sections will explain the muscle output response and muscle fitness results according to each stage of simulation.

\subsection{Single rectangular pulse}

Figure 5 shows the muscle activation, for a single rectangular stimulation pulse at different pulse widths $(100 \mu \mathrm{s}-500 \mu \mathrm{s})$ and frequencies $(20,30,50 \mathrm{~Hz})$. The muscle activation graph ranges between 0 and 1 , with value 1 represents full amount of activated muscle fibres and value 0 represents no measurable response to stimulation. At lower stimulation pulse widths, $100 \mu \mathrm{s}$ and $200 \mu \mathrm{s}$, the results indicate that there is no significant response of activated muscle even with varies frequencies. The muscle activation responses start to increase with greater stimulation pulse width. The overall results show that muscle activation differs with different stimulation frequencies as higher frequency causes greater muscle activation.

Stimulation pulse width of $500 \mu \mathrm{s}$ caused significant impact and produced big difference to the muscle activation as shown in Figure 5(e). Additionally, it is found that stimulation frequency of $50 \mathrm{~Hz}$ is able to optimize the activation of muscle fibres. The simulated results also indicate that higher stimulation frequency has greater effects to the reduction of muscle activation as the stimulation continues. It can also be seen from the slope of the graph that the higher the frequency, the more reduction in muscle activation, which also indicates that the muscle experience greater fatigue for higher frequency. The obtained results are in line with other researchers findings in muscle model output response [24-26]. 

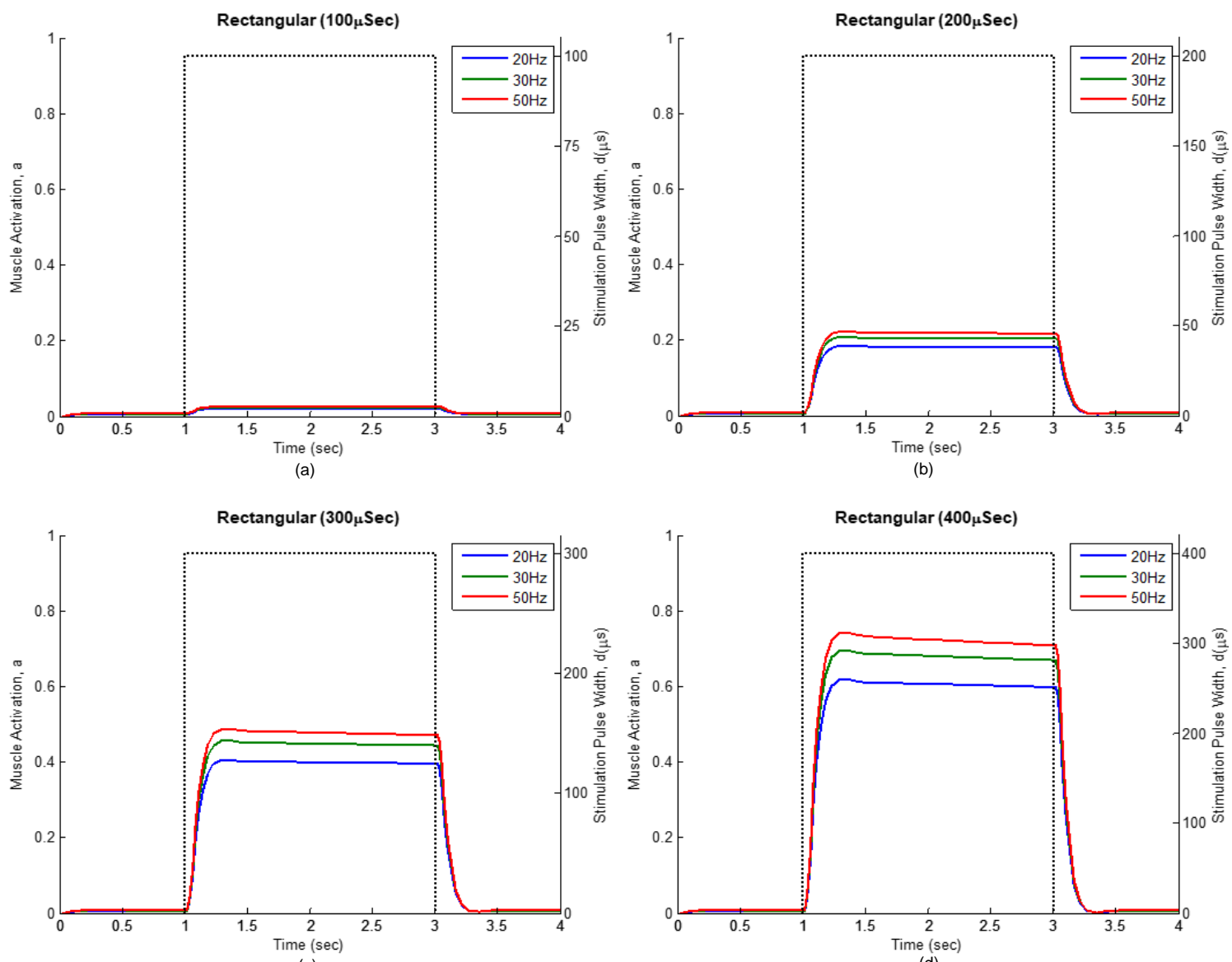

(c)

(d)

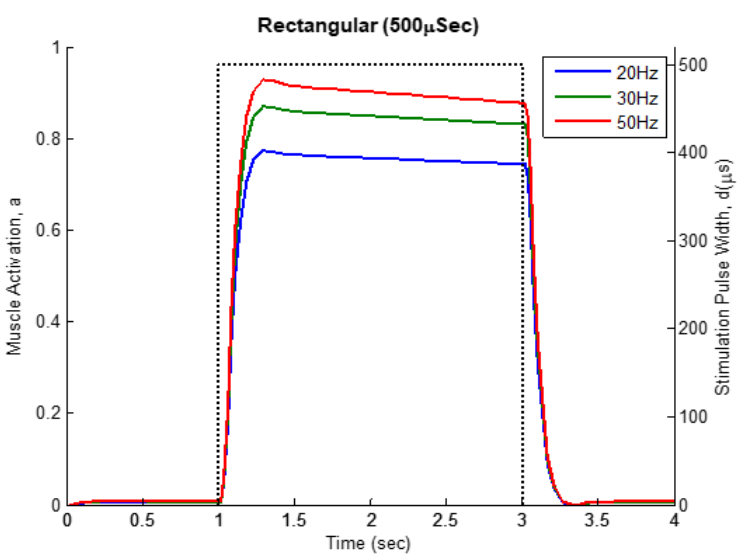

(e)

Figure 5. Rectangular stimulation waveform with 5 ranges of pulse widths $(100 \mu \mathrm{s}-500 \mu \mathrm{s})$ and 3 ranges of frequencies $(20 \mathrm{~Hz}, 30 \mathrm{~Hz}$ and $50 \mathrm{~Hz})$ for $4 \mathrm{~s}$ of stimulation period

\subsection{Pulse train of rectangular pulse}

In single Rectangular pulse stimulation, the effect of muscle fitness at different stimulation pulse widths could not be determined due to a short stimulation period (2s). Therefore, pulse train of rectangular stimulation at different pulse widths $(100 \mu \mathrm{s}-500 \mu \mathrm{s})$ and frequencies $(20 \mathrm{~Hz}, 30 \mathrm{~Hz}, 50 \mathrm{~Hz})$ were tested and evaluated. Figure 6 shows the muscle activation results, for pulse train of rectangular stimulation at different pulse widths and frequencies.

At lower stimulation pulse widths, $100 \mu$ s and $200 \mu$ s, the muscle activations are consistent for the whole 60s stimulation period with activation of 0.03 to 0.2 as observed in Figure 6(a) and 6(b). However, at higher stimulation pulse width, the muscle activation started to reduce as time increases. In Figure 6(c), 
with stimulation pulse width of $300 \mu \mathrm{s}$, the muscle activation reduced from 0.49 to 0.42 for the $60 \mathrm{~s}$ stimulation period. In Figure 6(d), with stimulation pulse width of $400 \mu \mathrm{s}$, the muscle activation reduced from

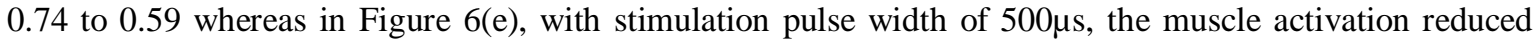
from 0.93 to 0.69 . Therefore, it can be concluded that higher stimulation pulse width resulted in higher reduction of activated muscle. Additionally, the muscle activation is also observed to increase as the stimulation frequency increases from $20 \mathrm{~Hz}$ to $30 \mathrm{~Hz}$. However, the muscle activation does not increase further as the stimulation frequency increases to $50 \mathrm{~Hz}$ after $40 \mathrm{~s}$ of stimulation period especially for higher stimulation pulse width. Therefore, for further muscle fatigue analyses, it is sufficient to use $30 \mathrm{~Hz}$ stimulation frequency and $500 \mu$ s pulse width for all types of flexible waveforms.
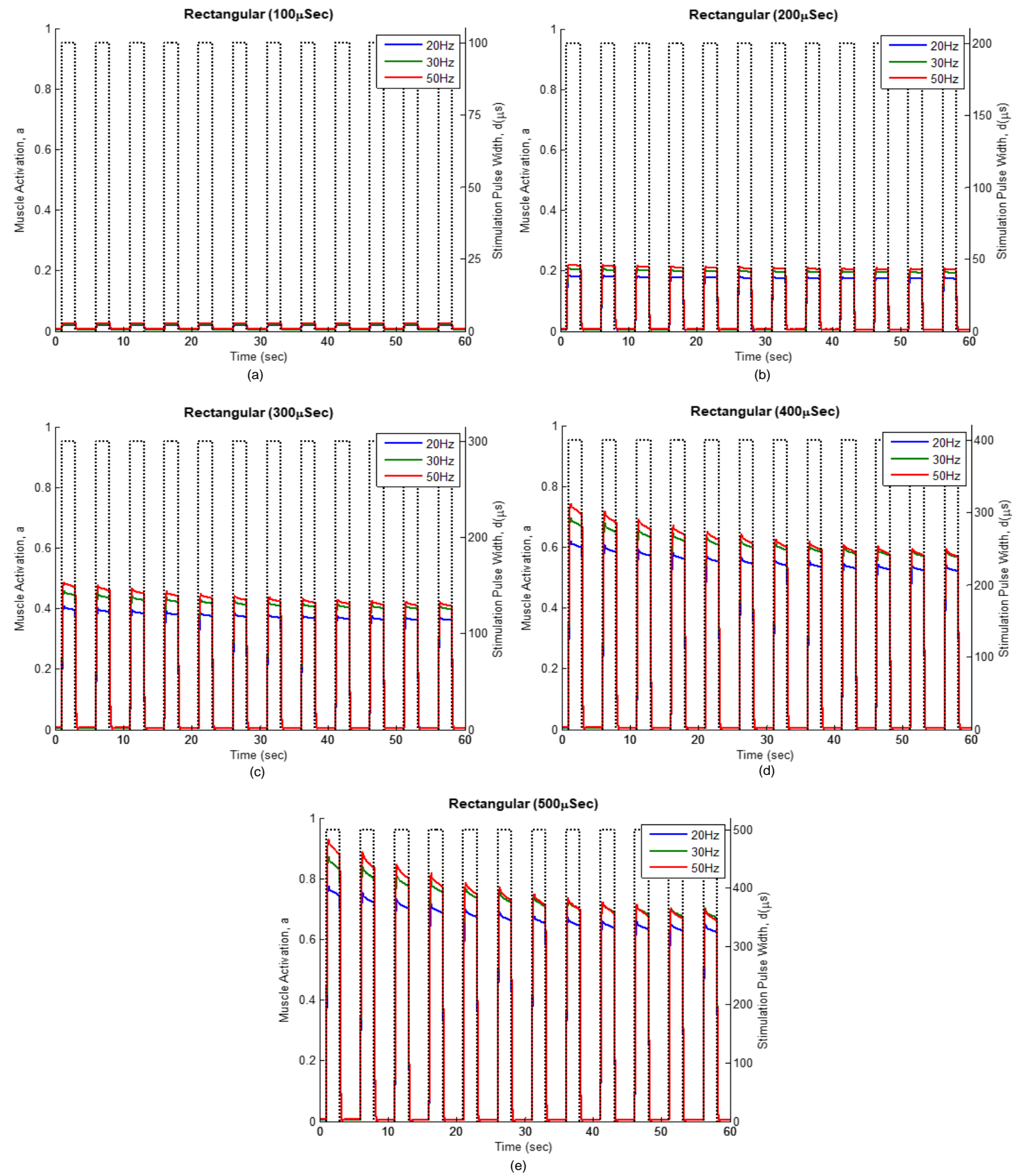

Figure 6. Pulse train of rectangular stimulation waveform with 5 ranges of pulse widths $(100 \mu s-500 \mu s)$ and 3 ranges of frequencies $(20 \mathrm{~Hz}, 30 \mathrm{~Hz}$ and $50 \mathrm{~Hz})$ for $60 \mathrm{~s}$ of stimulation period 


\subsection{Single flexible stimulation waveform}

Figure 7 depicts the muscle activation, for a single flexible stimulation waveform. As mentioned earlier, five different types of flexible stimulation waveforms (Rectangular, Trapezoidal, Ramp Up, Ramp Down and Triangular) were employed in this analysis of study. All five stimulation waveforms were generated by modulating the stimulation pulse width from $100 \mu \mathrm{s}-500 \mu \mathrm{s}$ at stimulation frequency of $30 \mathrm{~Hz}$. The muscle activation obtained by the Rectangular shape of stimulation was used as a reference to compare the performance of the other 4 types of stimulation waveforms.

Results shows some delay in the muscle activation response when Trapezoidal, Ramp Up and Triangular shapes of stimulation were applied at the muscle model. However, the Ramp Down and Rectangular shapes have shown only small delay in muscle activation response. It can also be observed that there are slight drops of muscle activation when the Ramp Up, Ramp Down and Triangular shape stimulation waveforms were applied.
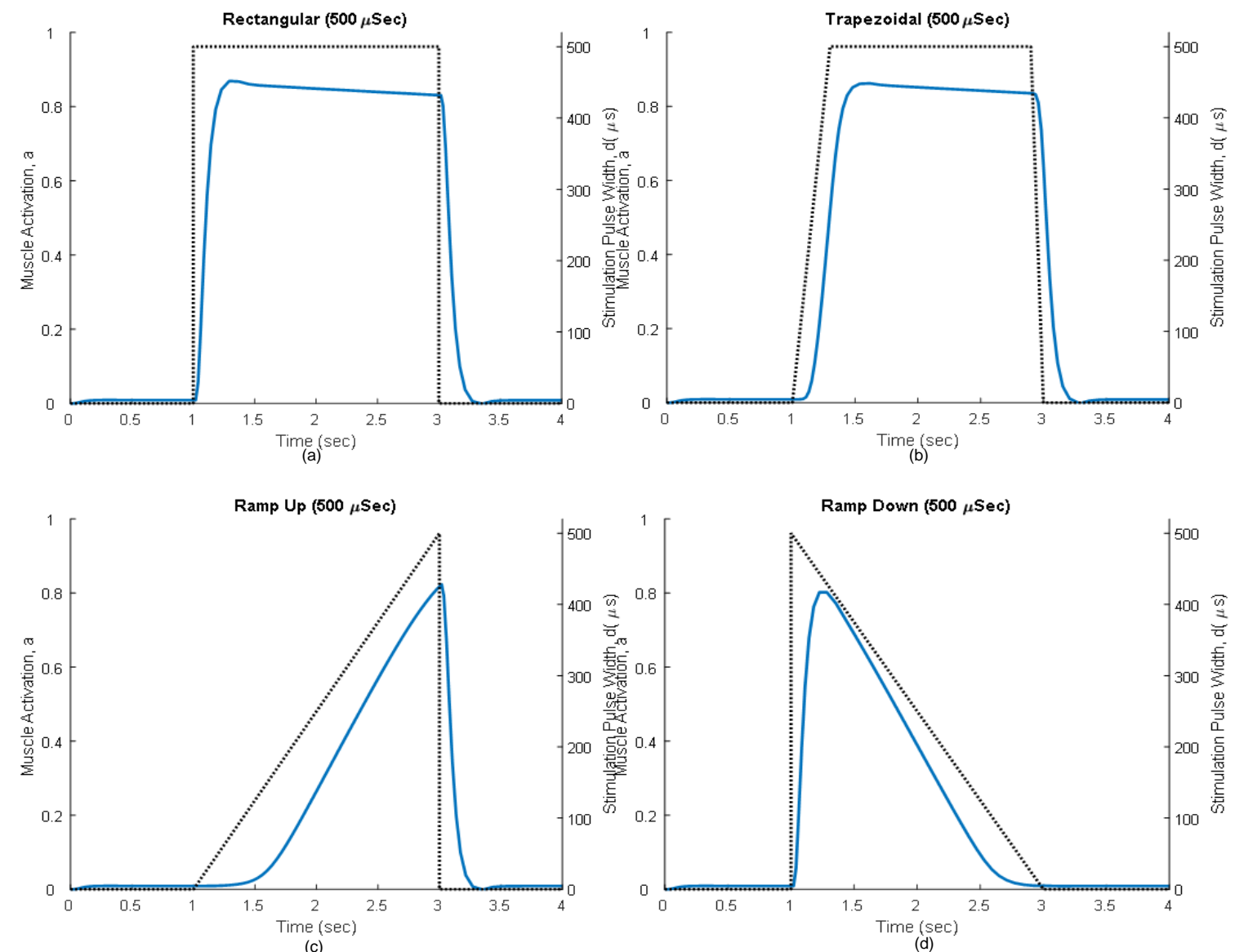

Triangular $(500 \mu \mathrm{Sec})$

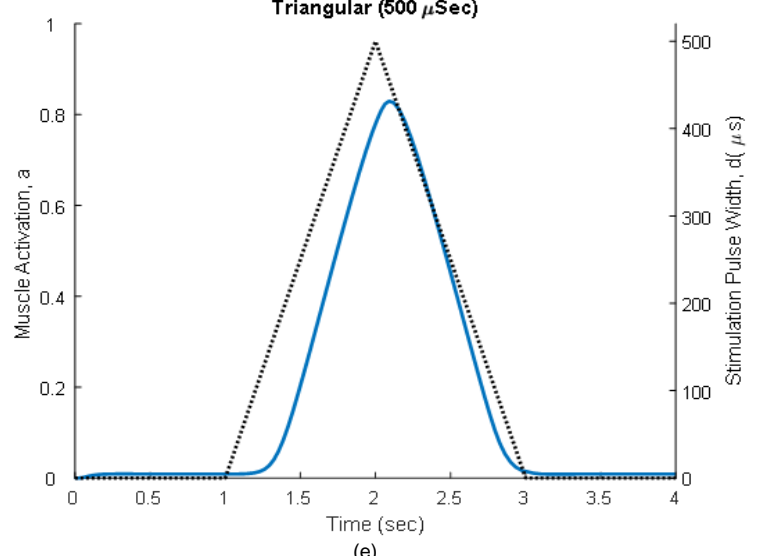

Figure 7. Single flexible stimulation waveforms at pulse width $=500 \mu$ s and frequency $=30 \mathrm{~Hz}$ 


\subsection{Pulse train of flexible stimulation waveform}

In this section the five different types of flexible stimulation waveforms (Rectangular, Trapezoidal, Ramp Up, Ramp Down and Triangular) were tested and simulated onto the muscle activation model for a duration of 60 s as shown in the Figure 8. Similar to the single flexible waveform, the stimulus parameters used were $500 \mu \mathrm{s}$ for pulse width and $30 \mathrm{~Hz}$ for stimulation frequency to characterize muscle activation and muscle fitness for $60 \mathrm{~s}$ of stimulation period. The effect of muscle fitness through the usage of different pulse train of flexible stimulation waveform were analysed. Figure 8 shows the muscle fitness of the five-pulse train of flexible stimulation waveforms for 60 s of stimulation period. Detail fitness values for all stimulation waveforms are tabulated in Table 2.

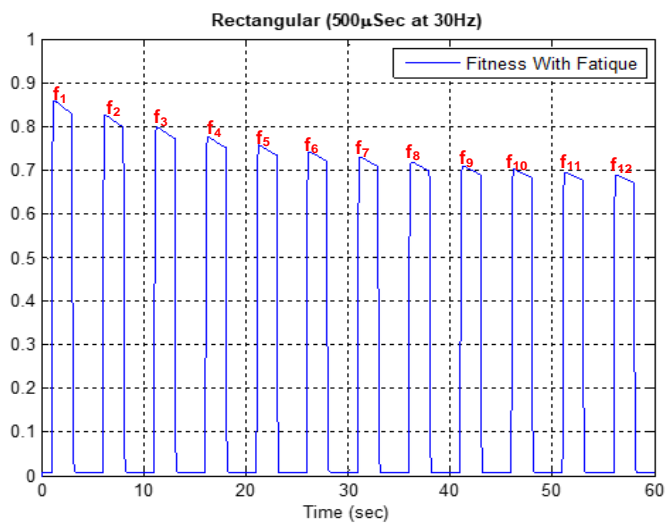

(a)

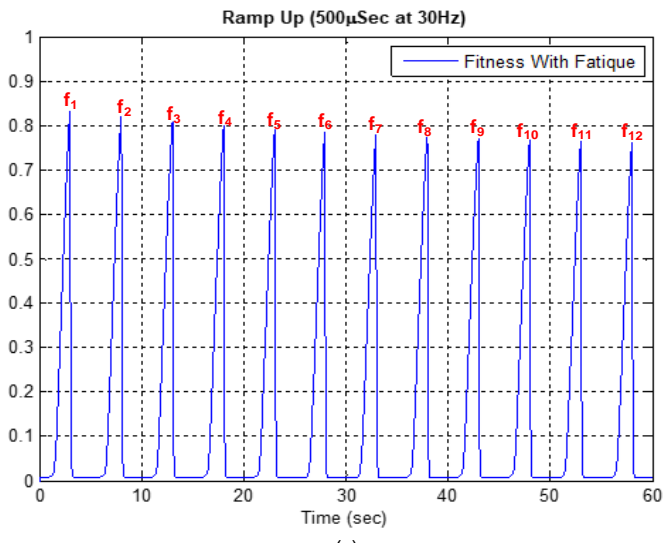

(c)

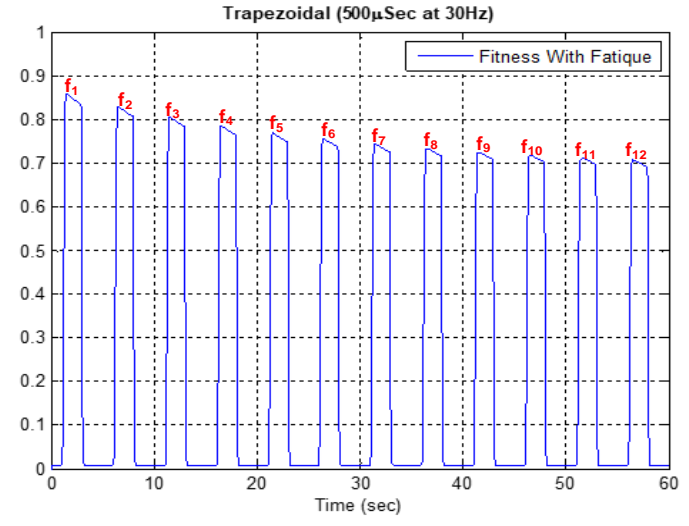

(b)

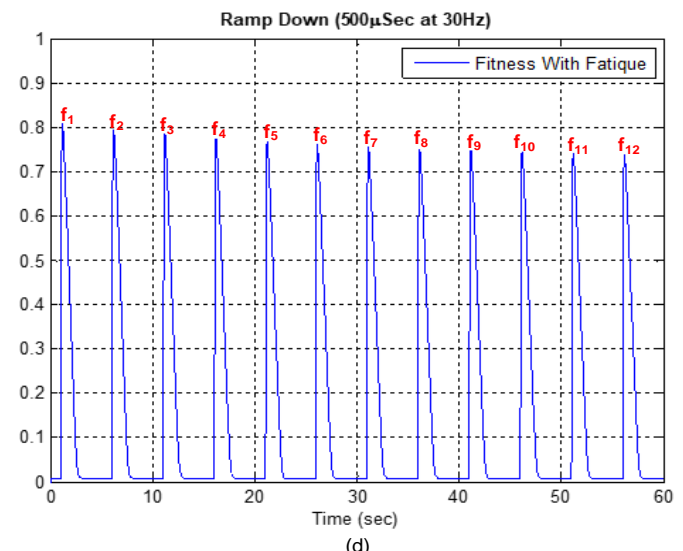

(d)

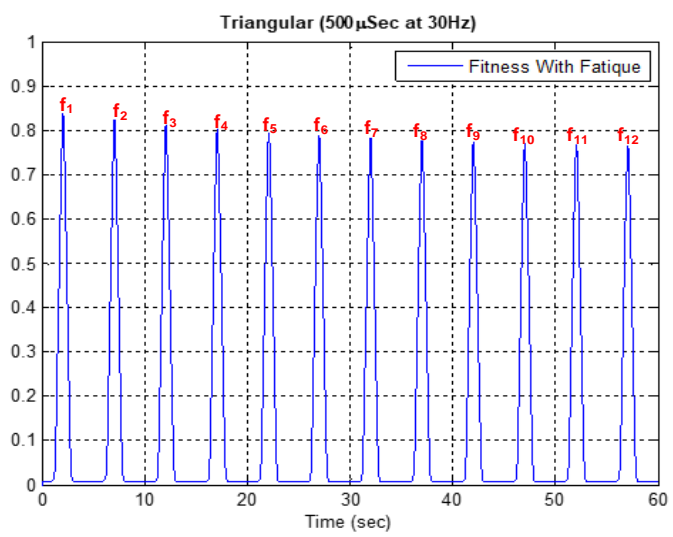

(e)

Figure 8. Pulse train of flexible stimulation waveforms at pulse width $=500 \mu \mathrm{s}$ and frequency $=30 \mathrm{~Hz}$ for 60 s stimulation 
For the first cycle of stimulation, muscle fitness is in the range of 0.859 to 0.807 . Rectangular and Trapezoidal shapes of flexible stimulation waveforms generate higher fitness level, whereas Ramp Down generates the lowest muscle fitness at 0.807 . Around 60 s of stimulation period, muscle fitness for all five types of flexible stimulation waveforms are reduced. The Rectangular shape of flexible stimulation waveform shows the greatest reduction of muscle fitness from 0.859 to 0.689 , which is then followed by Trapezoidal shape with reduction from 0.859 to 0.708 as shown in Figure 8(a) and 8(b), respectively. Triangular, Ramp Up and Ramp Down shapes show lower reduction of muscle fitness as depicted in Figure 8(c), 8(d) and 8(e). The reduction of muscle fitness for $60 \mathrm{~s}$ of stimulation period for these types of flexible stimulation waveforms are in the range of 0.068 to 0.070 and Ramp Down shape shows the lowest reduction of muscle activation from 0.8067 to 0.7383 .

\subsection{Fitness fatigue analyses}

The fitness analyses were further carried out to justify the fatigue profile of the muscle for a $60 \mathrm{~s}$ stimulation period using five different types of flexible stimulation waveforms at pulse widths of $500 \mu \mathrm{s}$ and frequency of $30 \mathrm{~Hz}$. At this point, negative slope calculations were carried out to evaluate the muscle fitness. As can be seen in the Table 2 , the muscle fitness profiles for the $1^{\text {st }}$ until $12^{\text {th }}$ cycle of 60 s stimulation period were measured for all five different types of stimulation waveforms. Table 3 lists the calculated values of average and max-min negative slopes for all five different types of stimulation waveforms. From the tabulated results in Table 3 , it is observed that the calculated values of average and max-min negative slopes are almost the same for all flexible stimulation waveforms. Therefore, for simplification, the analyses of muscle fatigue were referred to the average of negative slope values only.

The muscle fitness profiles as depicted in Figure 8, shows that the fitness levels are reduced over time and this lead to the increment of the negative slope magnitudes. The average of negative slope calculated for the Rectangular stimulation waveform was equal to 0.0031 . This is the highest negative slope among the five different types of flexible stimulation waveforms, which is then followed by Trapezoidal with 0.0027. The average negative slopes calculated for Triangular, Ramp Up and Ramp Down stimulation waveforms were equal to $0.0013,0.0013$ and 0.0012 , respectively. The higher negative slope magnitude indicates the reduction of muscle fitness and the increment of muscle fatigue. The Ramp Down shape shows the lowest average of negative slope, which indirectly indicate reduction in early muscle fatigue problem. On the contrary, the Rectangular shape of flexible stimulation waveform shows the highest average of negative slope, which indicate increment in the muscle fatigue problem.

Table 2. Muscle fitness for five types of flexible stimulation waveforms

\begin{tabular}{cccccc}
\hline Fitness & Rectangular & Trapezoidal & Ramp Up & Ramp Down & Triangular \\
\hline $\mathrm{f}_{1}$ & 0.8591 & 0.8592 & 0.8324 & 0.8067 & 0.8363 \\
$\mathrm{f}_{2}$ & 0.8273 & 0.8302 & 0.8192 & 0.7936 & 0.8162 \\
$\mathrm{f}_{3}$ & 0.7998 & 0.806 & 0.8081 & 0.7841 & 0.812 \\
$\mathrm{f}_{4}$ & 0.7761 & 0.7855 & 0.7988 & 0.7749 & 0.8025 \\
$\mathrm{f}_{5}$ & 0.7578 & 0.7678 & 0.791 & 0.7671 & 0.7946 \\
$\mathrm{f}_{6}$ & 0.7423 & 0.7549 & 0.7844 & 0.7605 & 0.7879 \\
$\mathrm{f}_{7}$ & 0.7292 & 0.7434 & 0.7789 & 0.7551 & 0.7823 \\
$\mathrm{f}_{8}$ & 0.7184 & 0.7337 & 0.7742 & 0.7505 & $\mathbf{0 . 7 7 7 6}$ \\
$\mathrm{f}_{9}$ & 0.7083 & 0.7252 & 0.7703 & 0.7452 & 0.7736 \\
$\mathrm{f}_{10}$ & 0.7015 & 0.7185 & 0.7671 & 0.7433 & 0.7703 \\
$\mathrm{f}_{11}$ & 0.6931 & 0.712 & 0.7643 & 0.7392 & 0.7675 \\
$\mathrm{f}_{12}$ & 0.6893 & 0.708 & 0.762 & 0.7383 & 0.7651 \\
\hline
\end{tabular}

Table 3. Average and max-min negative slopes for five types of flexible stimulation waveforms

\begin{tabular}{cccccc}
\hline Neg. Slope & Rectangular & Trapezoidal & Ramp Up & Ramp Down & Triangular \\
\hline $\mathrm{m}_{1}$ & 0.0063 & 0.0058 & 0.0026 & 0.0026 & 0.0040 \\
$\mathrm{~m}_{2}$ & 0.0055 & 0.0048 & 0.0022 & 0.0019 & 0.0008 \\
$\mathrm{~m}_{3}$ & 0.0048 & 0.0041 & 0.0019 & 0.0018 & 0.0019 \\
$\mathrm{~m}_{4}$ & 0.0036 & 0.0036 & 0.0016 & 0.0016 & 0.0016 \\
$\mathrm{~m}_{5}$ & 0.0031 & 0.0025 & 0.0013 & 0.0013 & 0.0013 \\
$\mathrm{~m}_{6}$ & 0.0026 & 0.0023 & 0.0011 & 0.0011 & 0.0011 \\
$\mathrm{~m}_{7}$ & 0.0022 & 0.0019 & 0.0009 & 0.0009 & 0.0009 \\
$\mathrm{~m}_{8}$ & 0.0020 & 0.0017 & 0.0008 & 0.0011 & 0.0008 \\
$\mathrm{~m}_{9}$ & 0.0014 & 0.0013 & 0.0006 & 0.0004 & 0.0007 \\
$\mathrm{~m}_{10}$ & 0.0017 & 0.0013 & 0.0006 & 0.0008 & 0.0006 \\
$\mathrm{~m}_{11}$ & 0.0008 & 0.0008 & 0.0005 & 0.0002 & 0.0005 \\
Average & 0.0031 & 0.0028 & 0.0013 & 0.0012 & 0.0013 \\
Max - Min $(\mathrm{ml}-\mathrm{m} 11)$ & 0.0031 & 0.0027 & 0.0013 & $\mathbf{0 . 0 0 1 2}$ & 0.0013 \\
\hline
\end{tabular}


Referring to the previous work by Riener et.al [25], using triangular stimulation waveform led to muscle fitness of approximately 0.665 (muscle fatigue of 34.5\%) after applying 40s continuous stimulation. With this new proposed stimulation strategy, the triangular waveform resulted in muscle fitness of 0.7776 (muscle fatigue of 22.24\%), after applying 40s continuous stimulation as highlighted in Table 2. Thus, the new proposed stimulation strategy with flexible stimulation waveform could reduce the problem early muscle fatigue phenomenon that exists in the FES device.

\section{CONCLUSION}

The analytical study of flexible stimulation waveform in muscle fatigue reduction have been explained and elucidated. Five types of flexible stimulation waveforms namely Rectangular, Trapezoidal, Ramp Up, Ramp Down, Triangular have been developed by varying the pulse width duration $(100 \mu \mathrm{s}-500 \mu \mathrm{s})$ and the stimulation frequency $(20 \mathrm{~Hz}, 30 \mathrm{~Hz}, 50 \mathrm{~Hz})$ using MATLAB/Simulink software. The generated waveforms were applied onto a humanoid muscle model to investigate the muscle output response and muscle fatigue reduction. The flexible stimulation waveforms were tested onto the humanoid muscle model in four different stages namely single Rectangular stimulation waveform, pulse train of Rectangular stimulation waveform, single flexible stimulation waveform and pulse train of flexible stimulation waveform to analyse the performance of each flexible stimulation waveform with respect to muscle output response and muscle fatigue. For single Rectangular waveform, the results indicated that there is no significant response of muscle activation for lower pulse width duration $(100 \mu \mathrm{s}$ and $200 \mu \mathrm{s})$. However, at higher pulse width, the muscle activation response started to increase. Additionally, the overall results also indicated that as frequency increases, the muscle activation response also increases. From output responses of pulse train Rectangular stimulation waveform, it was observed that muscle activation increased as the frequency increased from $20 \mathrm{~Hz}$ to $30 \mathrm{~Hz}$. However, further increment of frequency to $50 \mathrm{~Hz}$ did not increase the muscle activation response especially for higher pulse width $(500 \mu \mathrm{s})$ after $40 \mathrm{~s}$ of stimulation period. Therefore, for the next stages of stimulation testing, it was sufficient to use $30 \mathrm{~Hz}$ stimulation frequency and $500 \mu$ s pulse width for all types of flexible waveforms. From output responses of pulse train flexible stimulation waveforms, it was found that Ramp Down has the lowest muscle fitness reduction of 0.068 , while Rectangular shape has the highest muscle fitness reduction of 0.1698 . Additionally, it was observed that flexible stimulation waveforms of Ramp Down, Ramp Up and Triangular shapes have lower average of negative slopes in the range of 0.0012 to 0,0013 . The Rectangular and Trapezoidal shapes were observed to have higher negative slopes in the range of 0.0031 to 0.0028 . Among the flexible stimulation waveforms, it was found that the Ramp Down shape has the lowest average of negative slope, which indirectly led to highest muscle fatigue reduction. In contrast, the Rectangular shape has the highest average of negative slope, which indirectly led to the lowest muscle fatigue reduction. Therefore, from the conducted analyses, it can be concluded that the flexible stimulation waveforms such as Ramp Down, Ramp Up and Triangular shapes could reduce the early muscle fatigue problem, which is beneficial to the FES-assisted hemiplegic activities.

\section{ACKNOWLEDGEMENTS}

The authors would like to acknowledge Malaysian Ministry of Higher Education and UiTM for the FRGS grant (600-IRMI/FRGS 5/3 (046/2019)).

\section{REFERENCES}

[1] E. J. Gonzalez, et al., "Influence of Elbow Flexion and Stimulation Site on Neuromuscular Electrical Stimulation of the Biceps Brachii," IEEE Transactions on Neural Systems and Rehabilitation Engineering, vol. 26, pp. 904-910, 2018.

[2] L. P. Maneski, et al., "Assessment of Spasticity by a Pendulum Test in SCI Patients Who Exercise FES Cycling or Receive Only Conventional Therapy," IEEE Transactions on Neural Systems and Rehabilitation Engineering, vol. 26, pp. 181-187, 2018.

[3] H. Rouhani, et al., "Closed-loop control of standing neuroprosthesis using PID controller," 2017 IEEE International Conference on Systems, Man, and Cybernetics (SMC), pp. 2237-2242, 2017.

[4] D. Wolf, et al., "Combining functional electrical stimulation and a powered exoskeleton to control elbow flexion," 2017 International Symposium on Wearable Robotics and Rehabilitation (WeRob), pp. 1-2, 2017.

[5] V. D. Nguyen and Q. D. Chu, "Current generation circuit to functional electrical stimulate foot-drop patients," 2017 IEEE 4th International Conference on Smart Instrumentation, Measurement and Application, pp. 1-6, 2017.

[6] D. N. Rushton, "Functional electrical stimulation," J Physiological Measurement, vol. 18, pp. 241, 1997.

[7] W. M. Grill and J. T. Mortimer, "Stimulus waveforms for selective neural stimulation," J IEEE Eng. Med. Biol. Mag., vol. 14, pp. 375-385, 1995. 
[8] N. Alibeji, et al., "Dynamic surface control of neuromuscular electrical stimulation of a musculoskeletal system with activation dynamics and an input delay," American Control Conference (ACC), 2015, pp. 631-636, 2015.

[9] H. P. Wang, et al., "Design of a pulse-triggered four-channel functional electrical stimulator using complementary current source and time division multiplexing output method," Engineering in Medicine and Biology Society (EMBC), 2015 37th Annual International Conference of the IEEE, pp. 1671-1674, 2015.

[10] C. L. Lynch, M. R. Popovic, "Functional Electrical Stimulation," Control Systems, IEEE, vol. 28, pp. 40-50, 2008.

[11] C. S. To, et al., "Simulation of a functional neuromuscular stimulation powered mechanical gait orthosis with coordinated joint locking," Neural Systems and Rehabilitation Engineering, IEEE Transactions on, vol. 13, pp. 227-235, 2005.

[12] Z. Z. Karu, et al., "Reducing muscle fatigue in FES applications by stimulating with N-let pulse trains," Biomedical Engineering, IEEE Transactions on, vol. 42, pp. 809-817, 1995.

[13] G. M. Graham, et al., "The effect of random modulation of functional electrical stimulation parameters on muscle fatigue," Neural Systems and Rehabilitation Engineering, IEEE Transactions on, vol. 14, pp. 38-45, 2006.

[14] J. Naeem, et al., "An investigation of fatigue phenomenon in the upper limb muscle due to short duration pulses in an FES system," IOP Conference Series: Materials Science and Engineering, vol. 53, pp. 012067, 2013.

[15] P. Nadeau and M. Sawan, "A flexible high voltage biphasic current-controlled stimulator," IEEE Biomedical Circuits System Conference, BioCAS 2006, pp. 206-209, 2006.

[16] T. M. Bruns, et al., "Variable Patterned Pudendal Nerve Stimuli Improves Reflex Bladder Activation," Neural Systems and Rehabilitation Engineering, IEEE Transactions on, vol. 16, pp. 140-148, 2008.

[17] M. a. T. Sahin, Y, "Non-rectangular waveforms for neural stimulation with practical electrodes," J. Neural Eng., vol. 3, pp. 227, 2007.

[18] Z. P. Fang and J. T. Mortimer, "Selective activation of small motor axons by quasitrapezoidal current pulses," Biomedical Engineering, IEEE Transactions on, vol. 38, pp. 168-174, 1991.

[19] N. Accornero, et al., "Selective Activation of peripheral nerve fibre groups of different diameter by triangular shaped stimulus pulses," J Physiol, vol. 273, pp. 539-60, 1977.

[20] S. Jezernik and M. Morari, "Energy-optimal electrical excitation of nerve fibers," Biomedical Engineering, IEEE Transactions on, vol. 52, pp. 740-743, 2005.

[21] D. R. Cantrell and J. B. Troy, "Extracellular stimulation of mouse retinal ganglion cells with non-rectangular voltage-controlled waveforms," Engineering in Medicine and Biology Society, 2009. EMBC 2009. Annual International Conference of the IEEE, pp. 642-645, 2009.

[22] R. Riener and T. Fuhr, "Patient-driven control of FES-supported standing up: a simulation study," Rehabilitation Engineering, IEEE Transactions on, vol. 6, pp. 113-124, 1998.

[23] Z. Hussain, et al., "Muscle Extension Model for FES-Assisted Knee Swinging Ergometer for Stroke Patient," Computational Intelligence, Communication Systems and Networks (CICSyN), 2013 Fifth International Conference on, pp. 120-124, 2013.

[24] J. R. de Kroon, et al., "Relation between stimulation characteristics and clinical outcome in studies using electrical stimulation to improve motor control of the upper extremity in stroke," J Rehabil Med, vol. 37, pp. 65-74, 2005.

[25] R. Riener, et al., "Biomechanical model of the human knee evaluated by neuromuscular stimulation," J Biomech, vol. 29, pp. 1157-67, 1996.

[26] L. L. Baker, et al., "Effects of Waveform on Comfort During Neuromuscular Electrical Stimulation," Clinical Orthopaedics and Related Research ${ }^{\circledR}$, vol. 233, pp. 75-85, 1988.

\section{BIOGRAPHIES OF AUTHORS}

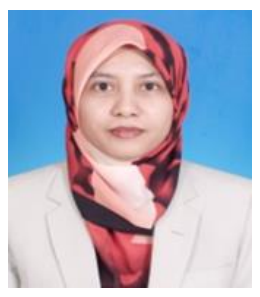

Emilia Noorsal is a senior lecturer at the Universiti Teknologi MARA Caw. Pulau Pinang. In April 2014, She obtained her PhD in biomedical engineering from Institute of Microelectronics, Ulm, Germany. Her research interests include digital design circuit in ASIC, FPGA, mixed-signal circuit design, power electronics and electronics for biomedical applications.

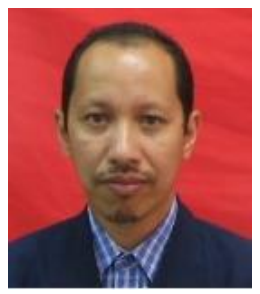

Mohammad Nizam Bin Ibrahim is a senior lecturer at the Universiti Teknologi MARA Caw. Pulau Pinang. He obtained his PhD in Power System Protection from University of Adelaide, South Australia in 2012. His research interests include power system protection simulation, faults analysis, numerical digital relay and Internet of Things. 


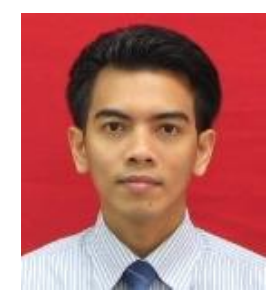

Saiful Zaimy Yahaya is a senior lecturer at the Universiti Teknologi MARA Caw. Pulau Pinang. $\mathrm{He}$ is currently pursuing his $\mathrm{PhD}$ in Intelligent Control Technique for Rehabilitation Engineering from Universiti Teknologi MARA Pulau Pinang. His research interests include Advanced Rehabilitation Engineering, Intelligent Control Techniques and Evolutionary Algorithms.

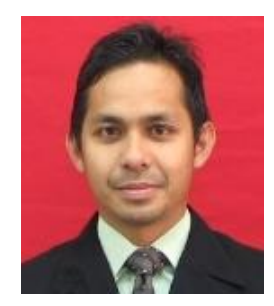

Rozan Boudville is a senior lecturer at the Universiti Teknologi MARA Caw. Pulau Pinang. $\mathrm{He}$ is currently pursuing his $\mathrm{PhD}$ in Intelligent Control Technique for Rehabilitation Engineering from Universiti Teknologi MARA Pulau Pinang. His research interests include Advanced Rehabilitation Engineering, Intelligent Control Techniques and Evolutionary Algorithms.

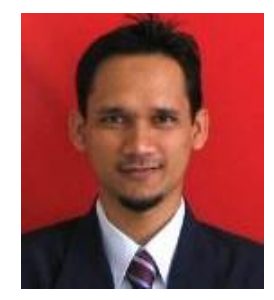

Zakaria Hussain is an Associate Professor at the Faculty of Electrical Engineering, Universiti Teknologi MARA Caw. Pulau Pinang. He obtained his PhD in Intelligent Control Technique for Rehabilitation Engineering from Automatic Control and System Engineering Department, the University of Sheffield, United Kingdom in 2010. His research interest includes Advanced Rehabilitation Engineering, Intelligent Control Techniques and Evolutionary Algorithms.

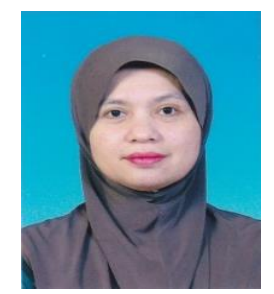

Yusnita Mohd Ali is a senior lecturer at the Faculty of Electrical Engineering, Universiti Teknologi MARA, Penang Campus, Malaysia. She received her PhD Degree in Mechatronic Engineering from Universiti Malaysia Perlis in 2014 specializing in Audio/Acoustic Engineering. She was conferred with a Master Degree in Electronics System Design Engineering from University Sains Malaysia in 2004. She completed her Bachelor Degree in Electrical \& Electronics Engineering from the same university in 1998. Her field of interest includes speech processing, speech analysis, human-machine interaction, brain-machine communication and artificial intelligence. 